\title{
Tras las huellas del aprendizaje significativo, lo alternativo y la innovación en el saber y la práctica pedagógica*
}

\author{
Alejandro Olaya' \\ Universidad Católica Lumen Gentium (Colombia) \\ Jackeline Ramírez ${ }^{2}$ \\ Secretaría de Educación Municipal de Santiago de Cali (Colombia)
}

Referencia formato APA: Olaya, A., \& Ramírez, J. (2015). Tras las huellas del aprendizaje significativo, lo alternativo y la innovación en el saber y la práctica pedagógica. Revista Científica Guillermo de Ockham, 13(2), 117-125.

\section{Resumen}

En este artículo se plantean las relaciones entre saber y práctica pedagógica. Estas acontecen como experiencia escolar que se expresa de múltiples maneras, entre ellas las experiencias que se denominan significativas, innovadoras y alternativas. En dicho sentido, se interroga aquellas prácticas educativas que se nombran así, a partir de una revisión documental con dos matrices que permiten analizar, primero, la forma como se configuran las experiencias desde los siguientes criterios: el proceso enseñanza-aprendizaje, el fin educativo que persiguen, sus estrategias didácticas y cómo se evalúa en las experiencias sistematizadas seleccionadas; y, en la segunda: cómo y dónde aparecen las categorías, qué función cumplen, instancias que le sirven de soporte, temas alrededor de los cuales se organizan y su interacción con otros conceptos. Del análisis de estas matrices en tales experiencias, se hallan sus sentidos, orígenes y formas de crear realidades y posicionamientos en la escuela y cómo en ella se configuran, reconfiguran y construyen discursos y prácticas pedagógicas.

Palabras clave: Innovación, aprendizaje significativo, alternativo, saber pedagógico y práctica pedagógica

\section{On the Traces of significant learning, alternative and innovation in knowledge and pedagogical practice}

\section{Abstract}

In this article the author presents the relations between knowledge and pedagogical practice. These occur as school experience is and is expressed in many ways, including the experiences that are called significant, innovative and / or alternatives. In that sense, those educational practices so named, from a literature review with two matrices that analyze, first, how the experiences are configured from the following criteria are questioning: the teaching-learning process, the educational purpose they pursue, their teaching strategies and how they evaluated the selected systematized experiences; and in the second: how and where categories appear, what role, instances that serve as support, themes around which they are organized and their interaction with other concepts. The analysis of these matrices, in such experiences, senses, origins and ways to create realities and positions in school and how it is set, reconfigure and build speeches and teaching practices.

Keywords: Innovation, significant learning, alternative, pedagogical knowledge and pedagogical practice

\footnotetext{
* Este artículo forma parte del proyecto Tras las huellas de la práctica y el saber pedagógico de las maestras y maestros en la expedición pedagógica nacional, del Proyecto Educativo y Pedagógico Alternativo y del Programa Ondas. Fecha de inicio: enero de 2013 y finaliza septiembre de 2014

1. Magíster en Educación: Desarrollo Humano. Teólogo de la Universidad Javeriana Bogotá. E-mail: aloar19@hotmail.com

2. Magíster en Educación: Desarrollo Humano. Licenciada en Ciencias Sociales. Tutora pedagógica y docente en el programa de transformación de la calidad educativa en la Secretaría de Educación Municipal de Cali. E-mail: jackerami@yahoo.com
} 


\section{Nas pegadas do aprendizagem significativa, alternativa e inovação do conhecimento e da prática pedagógica}

\section{Resumo}

Neste artigo surgir a relação entre conhecimento e prática pedagógica. Estes ocorrem como experiência escolar é e se expressa de várias maneiras, incluindo as experiências que são chamados significativa, inovadores e / ou alternativas. Nesse sentido, essas práticas educativas assim chamado, a partir de uma revisão da literatura com duas matrizes que analisar, em primeiro lugar, como as experiências são configurados a partir dos seguintes critérios estão questionando: o processo de ensino-aprendizagem, a finalidade educacional eles perseguem, suas estratégias de ensino e como eles avaliaram as experiências sistematizadas selecionados; e na segunda: como e onde aparecem categorias, qual o papel, os casos que servem de suporte, temas em torno dos quais eles são organizados e sua interação com outros conceitos. A análise destas matrizes, nas experiências, tais sentidos, origens e maneiras de criar realidades e as posiçóes na escola e como ela está definida, reconfigurar e construir discursos e práticas de ensino são.

Palavras-chave: Inovação, aprendizagem significativa, alternativa, conhecimento pedagógico e prática pedagógica

\section{Introducción}

La escuela, como un conjunto de discursos, estilos y formas de ser y hacer, se dibuja cada día como un entorno complejo, heteróclito y múltiple en sus diferentes esferas educativas, institucionales, comunitarias y subjetivas. La imagen canónica de la institución escolar, como santuario y representación de un orden fijo y normalizador, se torna anacrónica a pesar de los esfuerzos por sujetarla a un pasado y a un deber ser que día a día se transforma y cambia la fisionomía social y escolar de la institución. La escuela es un "cuadro vivo" que muchas veces se quiere ver inmóvil por los académicos, los políticos y los gobiernos de turno; pero al contrario, ella expresa un movimiento entre tradición y modernidad y entre lo evidente y lo emergente. Sin embargo, ¿cómo dar cuenta de dicho movimiento?

En este panorama, la búsqueda se centra en la posibilidad de leer el saber y la práctica pedagógica del maestro en las experiencias de la Expedición Pedagógica Nacional, el programa Ondas y el Proyecto Educativo Pedagógico Alternativo, para intentar hallar el modo como en estas experiencias se definen y enuncian en las prácticas de los maestros y maestras, reconociendo de esta manera el papel de control y vigilancia que tienen la institucionalidad estatal y los expertos del campo educativo.

En estas experiencias sistematizadas se ha hecho un rastreo que permite vislumbrar experiencias significativas, innovadoras y alternativas en los lugares donde se van desarrollando, sin que quienes las efectúan piensen o reflexionen sobre su propia experiencia. Cuando los maestros hablan de sus prácticas pedagógicas usan palabras como "significativo", "innovador" y "alternativo" con cierta ambigüedad conceptual que no permite pensar en una práctica de características precisas.

En esta exploración sobre las experiencias que se autonombran significativas, alternativas e innovadoras, sigue inquietando la pregunta por la constitución política del maestro que hace realidad estas experiencias alternativas, significativas o innovadoras en la educación y cómo su práctica es una acción política. Martínez-Pineda (2008) se propone "pensarlo y configurarlo como sujeto de acción política, capaz de actuar en instancias de definición de política educativa y de ejercer con fuerza la dimensión política que se le reclama a la educación y que se convierte en acción inherente a su profesión" (p. 18).

Responder estas inquietudes solo es posible, como lo menciona Tezanos (2007), "sometiendo a juicio riguroso, objetivo y sistemático que trascienda la opinión de los colegas" (p. 13) estas categorías; lo que implica poner en tensión y conflicto lo que se entiende en las prácticas pedagógicas por significativo, alternativo e innovador. $\mathrm{El}$ interés es indagar qué es lo significativo, innovador o alternativo y cómo estas categorías conceptuales se han transformado o utilizado de acuerdo con el contexto histórico y político en el ámbito educativo, puesto que el saber pedagógico es una práctica discursiva constituida en la escritura de la práctica de los maestros.

Finalmente, las inquietudes planteadas en este artículo están sometidas al efecto inapelable de la apropiación de Occidente de la producción del mundo "Este encuentro entre escuela, educación y medios de comunicación no está por fuera de las tensiones económicas y políticas que encarna el mundo del negocio mediático" (Rubio, 2011, p. 12), en el cual el modo de producción capitalista y sus 
leyes pretenden ser las únicas reconocidas como modo de desarrollo y progreso y por ende, la dependencia incondicional a los países y los capitales centrales que sustentan el poder desde las organizaciones de la banca mundial.

\section{Método}

En un primer paso se resuelven cuestiones inquietantes -cuando no sugestivas- para ser investigadas, como son las concepciones del mundo educativo sobre innovación, aprendizaje significativo y lo alternativo en la práctica pedagógica. Lo anterior está vinculado al hecho de usar categorías como puntos de partida, o en su defecto, de preceptos (manera de significación no analítica), y a partir de ellos dar cuenta de las rutas de exploración. Bien puede ser el caso de la migración conceptual, la emergencia o la pérdida de valor de rasgos singulares en la composición de sentido. Precisamente, estos elementos, a lo largo del trabajo investigativo, dan cuenta de la profundidad y de la manera de establecer relaciones en orden epistémico, entre ellas refiriendo categorías o momentos, horizontes o rasgos que en su interrelación sugieren rutas de sentido en las categorías ya mencionadas.

En un segundo momento, la ruta investigativa tomó la revisión documental como una forma de leer en las experiencias sistematizadas las prácticas pedagógicas de los maestros con los siguientes documentos: para la Expedición Pedagógica Nacional, el texto Expedición pedagógica nacional. Huellas y registros. Para el Programa Ondas, el texto revisado Informe de la reconstrucción colectiva del programa Ondas. Búsquedas de la investigación como estrategia pedagógica. Período 2006-2008. Y para el proyecto educativo y pedagógico alternativo (PEPA) el texto trabajado: Proyecto Educativo y pedagógico alternativo (2013). Una propuesta de Fecode para el país.

Con estos documentos se elaboraron dos matrices que permitieron el análisis de, en primer lugar, la forma como se configuran estas experiencias de acuerdo con los siguientes criterios: proceso enseńanza-aprendizaje, fin educativo que persiguen, estrategias didácticas y cómo se evalúa en las experiencias sistematizadas seleccionadas. En la segunda: cómo y dónde aparecen los términos significativo, alternativo e innovación; qué función cumplen, instancias que le sirven de soporte, temas alrededor de los cuales se organizan y su interacción con otros conceptos (o entre ellos) y las intencionalidades con que son construidos los discursos allí contenidos; es decir, "en una interpretación literal y la segunda, como una interpretación a partir de la reconstrucción del mundo del texto" (Sandoval, 1996, p. 68). Para ello se requieren algunas condiciones, como tener en cuenta la información relevante, disponible y elocuente para explicar el fenómeno interpretado a mediante una matriz de análisis construida para ello, en las que se identifican las lógicas de sentido de la utilización de las palabras significativo, innovador y alternativo.

\section{Resultados}

En coherencia con lo planteado en el método, los resultados tienen dos momentos: una búsqueda que permitió identificar el sentido, el origen y la evolución de las categorías aprendizaje significativo, innovación y alternativo, con las cuales, en un segundo momento, se hace la mirada y la discusión de las experiencias ya sistematizadas del PEPA, Ondas y Expedición Pedagógica.

\section{El sentido de las categorías}

\section{¿Aprendizaje significativo o experiencias significativas?}

El concepto aprendizaje significativo es mencionado por primera vez en 1963 por Ausubel y su teoría sobre psicología del aprendizaje verbal significativo, en la que diferencia entre aprendizaje y enseñanza y demuestra que la tarea esencial de la educación es conseguir el aprendizaje significativo. Propone el concepto para "distinguirlo del repetitivo o memorístico y señala el papel que juegan los conocimientos previos del alumno en la adquisición de nuevas informaciones. La significatividad solo es posible si se relacionan los nuevos conocimientos con los que ya posee el sujeto" (Nieda \& Macedo, 1997, p. 8).

El aprendizaje significativo ha sido interpretado de distintas maneras según el modelo pedagógico desde el cual se intente comprender. Es el caso de la escuela activa, cuya premisa es que la enseńanza por descubrimiento lo es de aprendizaje significativo, frene a lo cual De Zubiría (2006) explica que "lo significativo es el aprendizaje, no la enseñanza o método mediante el cual se da la enseñanza" (p. 125).

En los ańos sesenta, el constructivismo, como teoría pedagógica, toma lugar en el contexto educativo y se introduce el aprendizaje significativo de Ausubel. En este se tienen en cuenta los conocimientos previos que trae el niño a la escuela y a partir de ellos se reorganizan y anclan nuevos. Por lo tanto, lo significativo son los aprendizajes comprensibles y relevantes para el pensamiento y eso se logra mediante formas receptivas o por descubrimiento. 
"El aprendizaje por recepción y por descubrimiento puede ser repetitivo o significativo, según las condiciones en que ocurra el aprendizaje" (Ausubel, Novak \& Hanesian, 2001, p. 37).

A partir de la propuesta de Ausubel acerca de la importancia de entender y usar con sentido lo aprendido, la escuela de hoy señala situaciones, aprendizajes o enseñanzas de la vida diaria del entorno escolar como significativas para los estudiantes. Por ejemplo, cuando se observa en ellos un aprendizaje que nunca olvidarán o un determinado tema que despierta su interés de manera particular. Sin embargo, para el Ministerio de Educación Nacional (2009), el concepto de aprendizaje significativo se ha convertido en un asunto de experiencias significativas que comprende y propone como

Una práctica concreta (programa, proyecto, actividad) que nace en un ámbito educativo con el fin de desarrollar un aprendizaje significativo a través del fomento de las competencias; que se retroalimenta permanentemente a través de la autorreflexión crítica; es innovadora, atiende una necesidad del contexto identificada previamente, tiene una fundamentación teórica y metodológica coherente y genera impacto saludable en la calidad de vida de la comunidad en la cual está inmersa, posibilitando el mejoramiento continuo del establecimiento educativo en alguno o en todos sus componentes, tales como el académico, el directivo, el administrativo y el comunitario; fortaleciendo así, la calidad educativa (p. 10).

Esta definición está cargada de otras características más allá de lo planteado por Ausubel, en cuanto no se hace referencia al aprendizaje significativo como un logro del estudiante al incorporar un nuevo concepto a su conocimiento, sino a una experiencia que debe cumplir con unas características aportadas por otros actores en el proceso, y en este sentido ha trazado una "ruta del saber hacer" para que una experiencia significativa sea reconocida como tal.

Durante el 2007 el MEN diseñó un modelo conceptual de acompañamiento a las experiencias significativas orientado al fortalecimiento institucional (Colombia aprende, 2014). Por lo tanto, mientras el Ministerio en su función de mejorar la calidad de la educación intenta marcar la pauta para decidir qué es una experiencia significativa, acá se recurre a la comprensión propuesta por Ausubel que indica que son los aprendizajes y no las didácticas o las enseñanzas las que se califican como significativas.

\section{A qué se le llama alternativo}

Lo alternativo en el ámbito educativo se refiere a hacer visible lo no visible de las experiencias pedagógicas; visibi- lizar lo que nos están hablando esas prácticas más allá del discurso educativo oficializado y afrontar desde otras vías la cuestión educativa. Boaventura de Sousa (2011), propone que los conocimientos no científicos sean creíbles y aceptados como una práctica contrahegemónica. Es decir, no se desconoce el conocimiento científico, pero debe haber un diálogo en doble vía e "igualdad de oportunidades" entre estos y que no se naturalice lo llamado universal. Por lo tanto, no se desconocen las prácticas pedagógicas tradicionales, sino que se intenta reconocer otras posibles en igualdad de condiciones y oportunidades para reconocer su valor y aporte como forma de hacer, alternativa y legítima, que se diferencia de la forma universal y tradicional de la práctica pedagogía y la formación humana.

Para hacer frente a la contrarreforma educativa impulsada por el Gobierno de "hacer, pero reguladamente", los maestros continúan dando respuesta a estos imperativos mediante una resistencia hoy llamada "alternativa", para no ser asociados con política, izquierda, conflicto, contestatario, activista, pues quienes son identificados con estos adjetivos son perseguidos y señalados en el país para desaparecerlos o exterminarlos.

En los ochenta es posible percibir el perfeccionamiento del sistema de vigilancia y control pedagógico del Estado a través de la liberalización de la concepción de los usos "alternativos" de la práctica pedagógica. Lo que hasta los inicios de la década se inscribía en el campo de lo contestatario y de lo "alternativo" hacia finales de ella ha resultado familiarizado por el sistema (Díaz, 1991, p. 14).

Lo que empezó como resistencia se llamaría luego alternativo con el fin de quitarle el peso conflictivo, político, contestatario e izquierdista de la época. Dos décadas después y ante los señalamientos que se hacen en Colombia a quienes apuntan a lo alternativo en el discurso educativo, se transforma lo alternativo en innovación. Iniciativas que pueden ser alternativas se rotulan como innovadoras para ser visibilizadas y no ser marcadas como contestatarias o revolucionarias. Sin embargo, algunos continúan estos procesos en pequeños grupos para encontrarse, compartir y caminar en esas expectativas y compartirlas sin generar mucho ruido que luego los haga blanco de persecuciones y quizá de sospechas de locura; idealistas que, según los actores defensores del Estado docente, como lo llama (Álvarez, 2001), es necesario que trabajen realmente en los objetivos de la calidad.

Por lo tanto, lo alternativo se presenta como una posición política del maestro frente al mundo y sus contextos atestados de realidades dolorosas, crudas, desesperanzadoras y carentes de sentido por lo que se hace diariamente. 
Son las apuestas que los maestros hacen, conscientes de que a partir de la escuela se pueden hacer transformaciones que incidan en eso que todos sentimos y vivimos como una realidad que a veces sobrepasa las fuerzas. Por ello, lo que se llama alternativo está en la esfera de la discusión política pública para que no quede sujeto a la manipulación tecnocrática (Ratero, 2009). Pero además -según los rasgos que Aguilar (1991) propone para identificar experiencias pedagógicas alternativas- modificar sustancialmente el conjunto las relaciones escolares; es decir, aquellas entre los sujetos (en especial las relaciones de poder) y de estos con la cultura; debilitar las fronteras (muchas veces artificiales) de la escuela: la separación entre los saberes, la división de los tiempos y los espacios, la fragmentación de los ámbitos del desarrollo humano, la distancia entre la dinámica escolar y la del medio social; promover procesos de autogestión pedagógica y de transformación política que afectan la vida cotidiana de los involucrados en la búsqueda de la emancipación individual y colectiva. Ahora bien sin querer decir que estos son los únicos rasgos de alternatividad o que deben estar todos para que se llame alternativo.

\section{El concepto innovación}

Esta categoría aparece en los años sesenta "en las ciencias de la administración y se asocia a modernización" (Blanco-Guijarro \& Messina-Raimondi, 2000, p. 41), y es concebida como un proceso externo, definido y planeado por expertos. En el análisis de House (1988) acerca de las perspectivas para entender el surgimiento de la innovación educativa, liga indefectiblemente la idea de innovación con la noción de modernización:

Se supone que las cosas deben cambiar, que innovación significa progreso. EI proceso de modernización promete mejores condiciones materiales y una mayor realización individual. En concreto, la modernización es el paso de una realidad incontestable, que viene dada por la tradición, a una situación social en la que todos pueden cuestionarse y cambiarse. [...] Desde el punto de vista moderno, lo mismo puede "elegirse" en la industria o en la agricultura que en la educación (p. 24).

Lo anterior resalta el carácter histórico del término en cuanto la innovación depende de la sociedad y está en función del contexto cuyas demandas intenta satisfacer, al tiempo que se ve condicionada por posicionamientos políticos, sociales, culturales y epistemológicos (BlancoGuijarro \& Messina-Raimondi, 2000).

En la década de 1970, los estudios sobre innovación en Estados Unidos estaban dirigidos por tres perspectivas: la tecnológica, la política y la cultural. Estas perspectivas "constituyen el contexto conceptual en el que investigadores, tecnócratas y funcionarios comprendían el proceso de innovación, y proporcionaban el marco de referencia subyacente para la formulación de políticas" (House, 1988, p. 10).

Para la década de los noventa se retoma el concepto que inicialmente tuvo en 1960 en el área de la administración, con la perspectiva de calidad y competitividad en un mundo que se moderniza permanentemente. Este giro conceptual se mantiene hasta la actualidad y se fortalece en cuanto el movimiento pedagógico de los ochenta liderado por los maestros, se quedó en las reivindicaciones laborales del gremio y desatendió la discusión teórica y política de la pedagogía.

La innovación forma parte del aparato escolar para fortalecerlo y revitalizarlo. No obstante, se debe diferenciar una innovación de un simple cambio o mejora. "La categoría innovación tiene múltiples significados y su conceptualización está culturalmente condicionada, empleándose en muchos casos como una categoría 'de prestigio' para nombrar procesos que distan de ser innovadores" (BlancoGuijarro \& Messina-Raimondi, 2000, p. 21).

Por estas razones, el concepto de innovación requiere ser desarrollado y unificado en el mundo académico para tener una referencia que permita identificar cuándo se está frente a una experiencia innovadora con certeza (y no solo innovadora) para quienes hacen su sistematización. Se hace necesario diferenciar entre la teoría de la innovación y la práctica de la innovación, puesto que la práctica de la innovación es poco sistematizada o carece de una sistematización rigurosa que dé cuenta de todo su proceso. Además, las sistematizaciones existentes sobre prácticas pedagógicas innovadoras son, asimismo, sistematizadas por agentes externos a la innovación y no por sus protagonistas, sean estos los maestros o la comunidad.

Con todas las consideraciones anteriores y fundamentados más en las prácticas pedagógicas innovadoras que en el discurso de la innovación, se entiende que la innovación educativa retorna a las teorías administrativas de los años sesenta ligadas con la calidad y la competitividad, las cuales deciden qué es innovación a partir de discursos como las normas ISO 9000 y toda su estructura de gestión de calidad.

\section{Configuración de los discursos en las experiencias}

En la experiencia Ondas se hace referencia al aprendizaje significativo -como lo entendimos en esta obra-cuando 
el niño participa de la investigación y se hace un proceso para que anclen los conocimientos nuevos a partir de los anteriores. De hecho, aprovecha su contexto para generar un interés de indagación que lo lleve a aprender sobre determinada situación. "Construir experiencias significativas para los niños, las niñas y los jóvenes, a través de estrategias pedagógicas que los vinculen como actores centrales del proceso" (Ondas, 2009, p. 124).

En el marco del Plan Nacional de Educación de 1996, la Expedición Pedagógica es considerada una experiencia innovadora y en el portal Colombia Aprende -en la ruta del saber hacer- está ubicada como una experiencia significativa de los años noventa por cuanto es una forma de mostrar resultados y cumplir con sus funciones en este nivel. Sin embargo, para efectos del rastreo que se hizo en el texto Huellas y registros y con base en el referente ausbeliano, con toda seguridad en las experiencias de los docentes existió y existen este tipo de aprendizajes, pero no fue evidenciado en la sistematización que se hace de la EPN.

Por el contrario, a partir de esta búsqueda de los años noventa se organiza en el Ministerio un marco legal y procedimental para detectar y sistematizar estas experiencias. El portal Colombia Aprende tiene una de sus ventanas para este fin, pero no permite conocer las experiencias ya sistematizadas (Ministerio de Educación, 2009). Estos tienen un control absoluto y privativo del saber y del conocimiento que se generan en las experiencias significativas, como las nombra el Ministerio.

El PEPA recoge los pensamientos y bases de la educación liberadora, la educación popular y las pedagogías críticas y del pensamiento democrático en educación, a partir de autores como Paulo Freire, Peter McLaren, Henry Giroux, Michael Apple, Piedad Ortega, Antonio Gramsci, Pierre Bourdieu y Alain Touraine. Estos autores sirven de sustento teórico y conceptual y se inscriben en la pedagogía popular, crítica, marxista y transformadora para una sociedad pensada en términos de igualdad y justicia. El PEPA plantea esta construcción a partir de la lucha de clases, la construcción de una verdadera democracia y una nueva ética social que hagan frente a la explotación económica y la dominación cultural e ideológica de los pueblos, trascendiendo así desde la oposición contestataria a la práctica política propositiva, creativa y comprometida con las transformaciones estructurales de la desigualdad social, con miras a una sociedad incluyente en la diversidad, bajo unas relaciones objetivas y subjetivas de complementariedad y solidaridad (Fecode, 2012).

Por tanto, esta experiencia se organiza alrededor del saber y la experiencia del magisterio acerca de lo públi- co, enfrentando así la política educativa neoliberal y el conocimiento al servicio del poder, como una opción de resistencia a toda forma de enajenación humana y en la búsqueda de una educación de calidad. La resistencia, la movilización y la confrontación a partir del movimiento pedagógico, genera condiciones favorables para trazar una política alternativa que reivindique el valor histórico, social y cultural de la educación de los colombianos.

Su soporte principal lo configuran Fecode, como gremio que recoge y articula todo el magisterio colombiano; los CEID, como centros donde se impulsa la investigación docente; las comunidades académicas y educativas del país, y el movimiento pedagógico. La función que cumple esta categoría en el PEPA es incentivar a los maestros para que asuman su práctica y su saber pedagógico como una opción de lucha desde una posición político-pedagógica que se oriente a confrontar las políticas neoliberales con propuestas educativas diferentes, contrarias y alternas a lo que plantean el Ministerio y las tendencias pedagógicas que se inscriben en el ámbito de la dominación y explotación cultural en sus diversas manifestaciones.

Sin embargo, hasta el día de hoy los CEID, han avanzado - de forma muy solitaria- en la consolidación del proyecto y en el marco de la celebración de los treinta años del Movimiento Pedagógico en diciembre de 2012 publican el módulo del proyecto pedagógico, con unos contenidos más definidos y unas estrategias de trabajo más claras y aprobadas por resolución en la XIX asamblea general federal llevado a cabo en Paipa, Boyacá el 7 de marzo de 2013.

Hasta el momento falta dar a conocer a las bases del magisterio y demás integrantes de la comunidad educativa los avances logrados, a fin de recoger los aportes que sin duda habrán de hacer los docentes, estudiantes y padres de familia y enriquecer con tales insumos el proyecto, así como dar comienzo a un proceso de apropiación del proyecto por parte de las comunidades educativas en todo el país (Morales S. \& Ocampo 2009), acciones indispensables para que el PEPA se convierta en un objetivo nacional que se lograría mediante la búsqueda de reivindicaciones populares: la lucha unificada y decidida de las masas. En este sentido, Ávila (2009) enfatiza en la importancia en que el proceso del PEPA debe ser más importante que un programa de políticas con contenidos y aunque este debe existir, el proceso organizativo de lucha y comunidades académicas de saber pedagógico deben sustentarlo para permitir la defensa de la educación pública. Sin estos elementos, la lucha será infructuosa. 
Es importante enfatizar que la cuestión de lo alternativo -como tantas otras iniciativas de resistencia en cualquier campo social- tiende rápidamente a ser subsumida por el sistema a fin de invalidar o neutralizar los efectos que de ello se pudiera esperar. Por ello, el Ministerio retoma de las prácticas pedagógicas de resistencia, los "anhelos, necesidades y deseos de los maestros para producir cambios en el dispositivo" (Martínez \& Aguirre-Dávila, 2007, p. 75), como posibilidad de neutralizarlos. Por lo tanto, desde los años noventa se han llevado a cabo programas del Ministerio para capitalizar las prácticas de los maestros llamándolas experiencias significativas o innovadoras.

Todo aquello que era resistencia y alternatividad se tornó innovador para caber en el discurso institucional y quedar por fuera de seńalamientos. En el PEPA esta categoría retoma su significado beligerante y de resistencia de los años ochenta, incluso haciendo frente a lo que hemos categorizado en esta obra como innovación.

El PEPA asume la categoría de lo alternativo sin relacionar aun en su discurso lo significativo y la innovación. En esta medida, su opción por lo alternativo es clara y coherente, en cuanto sabe lo que implica el término, al tiempo que lo asume como campo en construcción de praxis política, social, cultural, pedagógica e ideológica.

La palabra alternativo es polisémica. Tanto en la política como en lo social tiene múltiples interpretaciones y prácticas, según las concepciones, intereses e historias de los diferentes sujetos sociales que actúan en la esfera de la resistencia. Como experiencia social y política, es un mapa que se extiende desde posiciones reformistas hasta enfoques revolucionarios y desde perspectivas nacionalistas hasta configuraciones internacionalistas (Fecode, 2012).

En Ondas, lo alternativo se menciona no como una categoría, sino como una palabra que impide homogenizar la propuesta y da lugar para discutir acerca de la diferencia étnica y cultural del país. En experiencias anteriores se menciona a Ondas y en ellas fue importante el aporte que instituciones educativas, ONG, gobiernos locales, universidades, empresas privadas y maestros, hicieron a la enseñanza de las ciencias con propuestas alternativas.

La innovación llega al país en los años sesenta y en los setenta y ochenta los maestros se apropian del concepto y desarrollan experiencias innovadoras. El programa Ondas es una vivencia pedagógica con trece años de existencia y ejecución. Nace por la necesidad de responder a un mandato constitucional y legal sobre ciencia, tecnología e innovación para la población colombiana.
La innovación tiene como función "proponer caminos alternativos en la construcción de una escuela que reconfigurada desde sus prácticas pedagógicas nos proponga transformaciones educativas para el siglo XXI". (Ondas, 2009, p. 10). Esta reconfiguración de la escuela debe estar acompañada de un asesor experto en investigación que les permita al estudiante y al maestro una experiencia grata en un asunto tan serio y riguroso como es la ciencia. Es decir, quien tiene el rigor del conocimiento y la investigación es el asesor y no el maestro.

El programa se vale de recursos teórico-prácticos para fortalecer su política de C+T e innovación. En sí mismo, se considera una propuesta innovadora a nivel pedagógico y busca la innovación en términos de producción técnica e instrumental, dejando así de lado las innovaciones pedagógicas que los maestros generan en esta práctica. En este sentido, el uso "indiscriminado" de las categorías innovación y alternativo no se traduce en desconocimiento, sino en una forma intencional de ordenar-normalizar el mundo educativo.

Las innovaciones y las alternativas tienen cabida en la escuela, pues como se mencionó anteriormente, el aparato escolar vive un proceso permanente de construcción social por medio del cual se produce la interacción de diversos procesos, a saber, reproducción, transformación, modernización, destrucción cultural, resistencia, refuncionalización de contenidos y formas escolares, uso y conservación de la memoria cultural, inculcación e imposición de pautas culturales, entre otros (Aguilar, 1991).

Una alternativa pedagógica es un constructo que se constituye en recurso heurístico para la interpretación de experiencias de cambio educativo. Propuesta ideológica que permita la condensación de un proyecto de transformación cultural [...] Una alternativa pedagógica se diferencia de una innovación en cuanto hace referencia a todas aquellas experiencias educativas que se diferencian de la práctica pedagógica tradicional y se oponen a ella. Pero a diferencia de los procesos de innovación educativa, no pretenden el mejoramiento del sistema educativo por la vía de la modernización, sino contribuir al logro de una nueva hegemonía política y cultural de las clases subalternas (pp. 57-58).

Lo alternativo tiene un "matiz político-ideológico donde la reflexión se ubica desde la resistencia y del conflicto frente a las prácticas hegemónicas culturales y sociales y no solo del poder económico" (Barrantes 2013, p. 18).

La expedición pedagógica no se pregunta por la categoría innovación, pero sí formula gran cantidad de preguntas alrededor de prácticas innovadoras. En el campo de las prácticas pedagógicas, se orienta por preguntar alrededor 
de la función que cumplen los diversos actores en la escuela y alrededor de sus prácticas pedagógicas (Expedición Pedagógica Nacional, 2001).

\section{Discusión}

\section{Discurso, saber y practica pedagógica}

El saber y la práctica, en cuanto se dan en la experiencia escolar, se expresan de múltiples maneras, entre ellas las tres mencionadas anteriormente (significativas, innovadoras y alternativas). En las experiencias educativas, el saber y la práctica van de la mano y aunque diferentes en su comprensión, su entramado de sentidos no se entiende el uno sin el otro. Sostienen una relación intrínseca entre la función del discurso y su necesario volcamiento en una práctica donde este adquiere sentido.

En el orden del discurso, Foucault (1970), plantea que la producción de los discursos es controlada, seleccionada y distribuida a partir de ciertos procedimientos, para nombrar la realidad con base en cierta institucionalidad o sistema de instituciones, que la imponen y le dan vigencia, creando así una forma de exclusión o separación históricamente constituida. Esta relación del poder y el deseo con el discurso, demuestra que los discursos no están libres de los intentos del poder de poner bajo su dominio los aspectos que caen en su ámbito y conllevan los procedimientos de prohibición que expone Foucault. Como institución, la educación forma parte de ese sustento de la voluntad de verdad que propone el autor:

La educación, por más que sea, de derecho, el instrumento gracias al cual todo individuo en una sociedad como la nuestra puede acceder a no importa qué tipo de discurso, se sabe que sigue en su distribución, en lo que permite y en lo que impide, las líneas que le vienen marcadas por las distancias, las oposiciones y las luchas sociales. Todo sistema de educación es una forma política de mantener o de modificar la adecuación de los discursos, con los saberes y los poderes que implican (Foucault, 1970, p. 38).

Al hilo de estos planteamientos, es pertinente indagar por el manejo de los discursos que en el mundo educativo recurren a categorías como lo significativo, lo alternativo y la innovación, para plantear la necesidad de investigar qué hay más allá de los significados aparentes y develar aquello que dentro de esos discursos se cruza, se yuxtapone, se ignora o se excluye, e intentar mostrar "cómo se han formado, responder a qué necesidades, cómo se han modificado y desplazado, qué coacción han ejercido efectivamente, en qué medida se han alterado" (Foucault, 1970, p. 38).

A partir del rastreo de estas concepciones y de la forma cómo se han desarrollado estas tres categorías en experiencias sistematizadas, es que se leen las prácticas de las maestras/maestros, su saber pedagógico y cómo éstas muestran que "lo nuevo no está en lo que se dice, sino en el acontecimiento de su retorno" (Foucault, 1970, p. 25), y la manera como estas se pronuncian y legitiman sin que quienes las usan en sus prácticas diarias necesariamente conozcan lo que cada una en realidad significan y construyen desde el discurso. Es decir, a partir de esta ruta epistémica, se propuso acercarse a cada una de estas categorías a partir de los discursos que las han sistematizado, enunciado y adjetivado.

Es en la práctica de los maestros que se hallan las lógicas y el sustento de los saberes pedagógicos. Ellos dan cuenta de su historia, de los fines educativos, de las relaciones entre los distintos temas y de las metodologías y conceptos usados. Es en el proceso de enseñanza-aprendizaje, en la implementación de las estrategias didácticas y en la forma como se evalúa, que se ponen en juego los conceptos, sin darse cuenta qué función cumplen, qué instancias le sirven de soporte, los temas alrededor de los cuales se organizan y su interacción entre ellos y otros conceptos. En este sentido, Zuluaga (2005) propone que "es el maestro el designado en la historia como el soporte del saber pedagógico" (p. 49). Es en estos espacios de práctica del saber la voz del maestro ha estado y está presente, aunque se intente desconocer sus aportes como sujeto productor de conocimiento de la educación y de las ciencias humanas.

El saber pedagógico permite analizar los diferentes elementos en torno a los cuales giran los discursos y las prácticas y comprender tanto aquellos que se insertan en la práctica, como los que se van produciendo en su transcurrir. A partir de este, mirar a los sujetos (maestros y otros profesionales que hoy participan de la educación) que se refieren a los objetos del saber (la enseńanza, el niño, la escuela, el conocimiento, la instrucción, el sujeto, los métodos de enseñanza, la relación ciencia enseñanza), y utilizan conceptos, métodos y procedimientos en sus acciones prácticas.

El saber pedagógico es el funcionamiento no científico de la pedagogía, lo cual quiere decir que lo que hace que la pedagogía funcione, exista, intervenga en una sociedad, no es la ciencia sino otra serie de fuerzas, formas, hechos y prácticas (Zuluaga, Echeverri, Martínez, Quiceno, Sáenz \& Álvarez, 2003, p. 12). 
Se comprende, entonces, el sentido del quehacer diario del maestro en el que involucra su humanidad y su conocimiento para obtener los resultados que se propone conscientemente con sus estudiantes y seres humanos a cargo. En la práctica pedagógica, se ponen en juego su saber, el saber de su vida y sus experiencias y no solo el conocimiento adquirido formalmente en la academia. Esa práctica pedagógica tiene todo un saber y con él se evidencia y analiza más allá de lo que plantea, permitiendo así develar la propuesta intrínseca en la práctica pedagógica sobre lo significativo, lo alternativo y lo innovador.

Las categorías significativo, innovación y alternativo, usadas en el caso de las experiencias analizadas, están interrelacionadas, diferenciadas, superpuestas, mezcladas, yuxtapuestas, en oposición, o como postergación unas de las otras, con la intención de ordenar el discurso y confirmar con Boaventura de Sousa que "los objetos tienen fronteras cada vez menos definidas; están constituidos por anillos que se entrecruzan en telas complejas con los restantes objetos, hasta tal punto que los objetos en sí son menos reales que las relaciones entre ellos" (Boaventura de Sousa, 2003, p. 81).

\section{Referencias}

Aguilar, J. (1991). La transformación de la escuela en Colombia. De las innovaciones educativas a las alternativas pedagógicas. Santa Fe de Bogotá: Cepecs.

Álvarez, A. (2001). Del Estado docente a la sociedad educadora: ¿un cambio de época?. Revista Iberoamericana de Educación, 26, 35-58.

Ausubel, D., Novak , J., \& Hanesian, H. (2001). Psicología educativa. Un punto de vista. México: Trillas.

Blanco-Guijarro, R., \& Messina-Raimondi, G. (2000). Estado del arte sobre innovaciones educativas en América Latina. Santiago de Chile: Convenio Andrés Bello.

Boaventura de Sousa, S. (2011). Hacia una sociología de las ausencias y una sociología de las emergencias, en una epistemología del sur. La reinvención del conocimiento y la emancipación social. México: Siglo XXI.

Boaventura de Sousa, S. (2003). Critica de la razón indolente. De la ciencia moderna al Nuevo Sentido Común. Bilbao: Desclëe de Brouwer.
Colombia aprende. (2014). Las rutas del saber hacer. Experiencias significativas. Recuperado de http://goo.gl/7T85DP

De Zubiría, J. (2006). Los modelos pedagógicos. Hacía una pedagogía dialogante. Bogotá: Magisterio.

Díaz, M. (1991). De la práctica pedagógica al texto pedagógico. Pedagogía y Saberes, 1, 14-27.

Foucault, M. (1970). El Orden del Discurso. Buenos Aires: Letra e.

Zuluaga, O. L., Echeverri, A., Martínez, A., Quiceno H., Sáenz, J., \& Álvarez, A. (2003). Pedagogía y Epistemología. Bogotá: Cooperativa Editorial Magisterio.

House, E. (1988). Tres perspectivas de la innovación educativa: tecnológica, política y cultural. Revista de Educación, 286, 5-84.

Martínez, E., \& Aguirre-Dávila. (2007). Investigación de los saberes pedagógicos. Bogotá: Ministerio de Educación Nacional.

Martínez-Pineda, M. C. (2008). Redes pedagógicas: la constitución del maestro como sujeto político. Bogotá: Magisterio.

Ministerio de Educación Nacional. (2009). Las rutas del saber hacer. Experiencias significativas que transforman la vida escolar. Bogotá: Ministerio de Educación Nacional.

Nieda, J., \& Macedo, B. (1997). Un currículo cientifico para estudiantes de 11-14 años. Madrid: UNESCO.

Ocampo T. J. F. (1999). La Educación Pública Colombiana: 1950-2000. Educación y Cultura, 50, 44-58.

Ratero, C. (2009). La pedagogía por inventar. En C. Skliar \& J. Larrosa, Experiencia y alteridad en educación (pp. 166-188). Santa Fe Argentina: Homo Sapiens.

Rubio, J. (2011). Educaciones y tecnicidad: Relaciones y tensiones (actuales) en el mundo educativo. Perspectivas, 1-27.

Sandoval, C. A. (1996). Investigación Cualitativa. Bogotá: ICFES.

Tezanos, A. (2007). Oficio de enseñar - saber pedagógico: la relación fundante. Educación y Ciudad, 12, 10-25.

Zuluaga, O. L. (2005). Foucault: Una lectura desde la práctica pedagógica. En O. L. Zuluaga et al., (Eds.), Foucault, la pedagogía y la Educación. Pensar de otro modo (pp. 71-104). Bogotá: Cooperativa Editorial Magisterio. 